\title{
An analysis of patient self-reported COVID-19 symptoms during the first wave of the pandemic in Ireland
}

\author{
Claire Gibbons $^{1}$ [D $\cdot$ Mouzzam Hussain ${ }^{1} \cdot$ Derek T. O'Keeffe ${ }^{2} \cdot$ Andrew J. Simpkin ${ }^{2}$
}

Received: 5 March 2021 / Accepted: 9 March 2021 / Published online: 25 March 2021

(c) Royal Academy of Medicine in Ireland 2021

\begin{abstract}
Background Since the outbreak of COVID-19 in December 2019, there have been more than 115 million cases worldwide (1). Symptoms of COVID-19 vary widely and the spectrum of clinical presentation has yet to be fully characterised (2). Many countries have detailed their early experience with COVID-19, with a focus on the clinical characteristics of the disease. However, to our knowledge, there has been no such study detailing symptoms in the Irish population.

Aim Our aim is to describe COVID-19 symptoms in the Irish population at the beginning of the COVID-19 pandemic and compare symptoms between those reporting positive and negative test results.

Method A Web page MyCovidSymptoms.ie was created by researchers at the National University of Ireland, Galway, in April 2020 to investigate COVID-19 symptoms in Ireland. The Web page invited participants to self-report RT-PCR test outcome data (positive, negative, untested), temperature and a range of symptoms (cough, shortness of breath, fatigue, loss of taste, loss of smell).

Results One hundred and twenty-three Irish participants who had a RT-PCR test for COVID-19 logged their symptoms. Eighty-four patients reported that they tested positive for COVID-19, and 39 patients reported a negative COVID-19 test. In our cohort of respondents with a positive COVID-19 test, 49/84 (58\%) respondents reported a cough. Of the 39 respondents with a negative COVID-19 test, 17 (44\%) reported having a cough. The distribution of temperature was similar in both those with and without COVID-19. Levels of self-reported fatigue were high in both groups with 65/84 (77\%) of COVID19-positive patients reporting fatigue and 30/39 (77\%) of those who were COVID-19-negative reporting fatigue. New symptoms emerging at the time of data collection included loss of taste and smell. We demonstrated a higher proportion of loss of smell $(p=0.02)$ and taste $(p=0.01)$ in those reporting a positive result, compared to those reporting a negative result. Conclusion These data represents an early picture of the clinical characteristics of COVID-19 in an Irish population. It also highlights the potential use of self-reported data globally as a powerful tool in helping with the pandemic.
\end{abstract}

Keywords COVID-19 $\cdot$ Clinical characteristics $\cdot$ Digital Health $\cdot$ Innovation $\cdot$ Symptoms

\section{Introduction}

Since the outbreak of COVID-19 in December 2019, there have been more than 115 million cases worldwide [1]. The first case of COVID-19 was notified in Ireland on

Claire Gibbons

clairegibbons2017@gmail.com

1 School of Medicine, College of Medicine, Nursing and Health Sciences, National University of Ireland, Galway, Ireland

2 School of Mathematics, Statistics and Applied Mathematics, National University of Ireland, Galway, Ireland
February 29, 2020, and as of March 1, 2021, Ireland has had $>220,000$ cases [1, 2]. Symptoms of COVID-19 vary widely, and the spectrum of clinical presentation has yet to be fully characterised [3].

With the potential for further emergence of global pandemics, the challenge is early, accurate identification of the disease and effective therapeutics. The COVID-19 pandemic has considerably outpaced our collective efforts to characterise the clinical presentation and identify those at risk of more serious sequelae [4]. In response, we have seen the emergence of technology that gathers actionable data on a large scale to expedite our response to a fast-moving global health crisis [4]. 
Many countries have detailed their early experience with COVID-19, with a focus on the clinical characteristics of the disease. However, to our knowledge, there has been no such study detailing symptoms in the Irish population.

\section{Aim}

Our aim is to describe COVID-19 symptoms in the Irish population at the beginning of the COVID-19 pandemic, and compare symptoms between those reporting positive and negative test results.

\section{Methods}

A Web page MyCovidSymptoms.ie was created by researchers at the National University of Ireland, Galway in April 2020 to investigate COVID-19 symptoms in Ireland. The Web page invited participants to self-report RT-PCR test outcome data (positive, negative, untested), temperature, a range of symptoms (cough, shortness of breath, fatigue, loss of taste, loss of smell) and their county location. No personal data (e.g. name, age) were collected.

\section{Statistical analysis}

In this report, we compare symptoms between those reporting a positive and negative result. A Wilcoxon rank sum test was used to compare temperature, and a Fisher's exact test was used to compare positive and negative participants across the symptoms of cough, shortness of breath, fatigue, loss of taste and loss of smell.

\section{Results}

One hundred and twenty-three Irish participants who had a RT-PCR test for COVID-19 logged their symptoms. Eighty-four patients reported that they tested positive for COVID-19, and 39 patients reported a negative COVID-19 test (Table 1).

\section{Temperature}

The mean reported temperature of those who reported testing positive for COVID-19 $(n=84)$ was $37.4{ }^{\circ} \mathrm{C}$. Among those reporting a negative result, the mean temperature was $37.8^{\circ} \mathrm{C}$. The distribution of temperature was similar in both those with and without COVID-19 (Fig. 1).

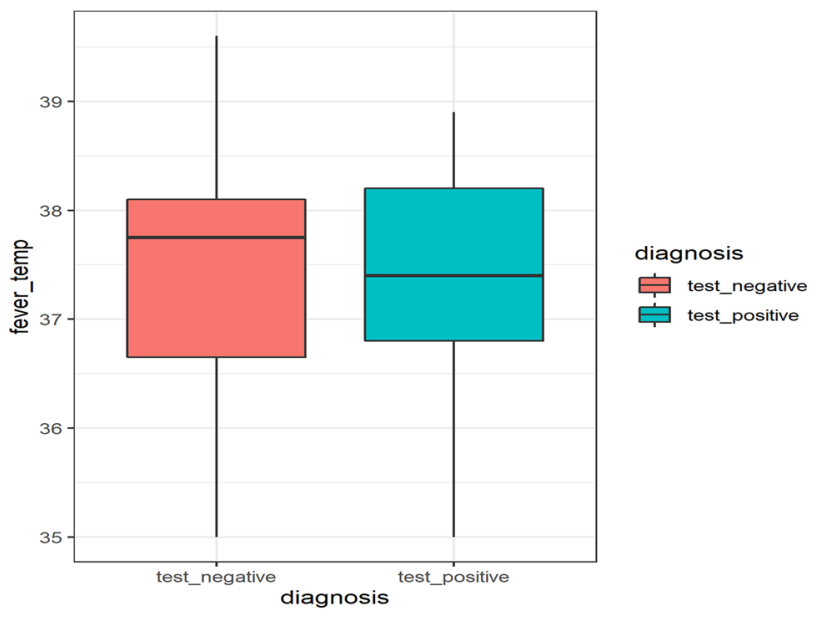

Fig. 1 Boxplot of temperature among COVID-19-positive and COVID-19-negative respondents

\section{Cough}

In our cohort of respondents with a positive COVID-19 test, 49/84 (58\%) respondents reported a cough. Of the 39 respondents with a negative COVID-19 test, 17 (44\%) reported having a cough (Fig. 2).

\section{Shortness of breath}

In the cohort of respondents with a positive COVID-19 test, 31/84 (37\%) reported shortness of breath, whereas $11 / 39$ (28\%) of those with a negative test reported shortness of breath (Fig. 3).

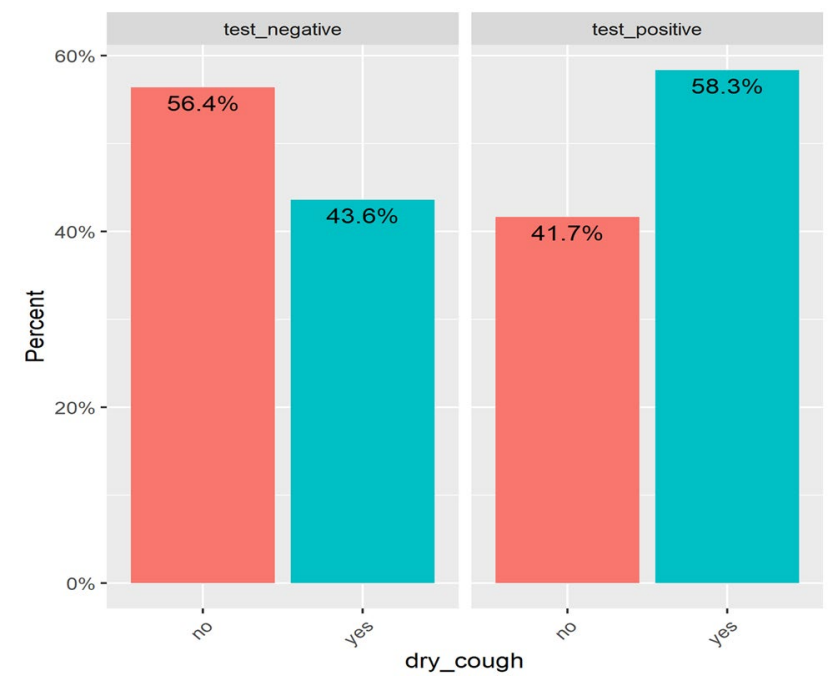

Fig. 2 Self-reported cough in respondents with a positive and negative COVID-19 test 


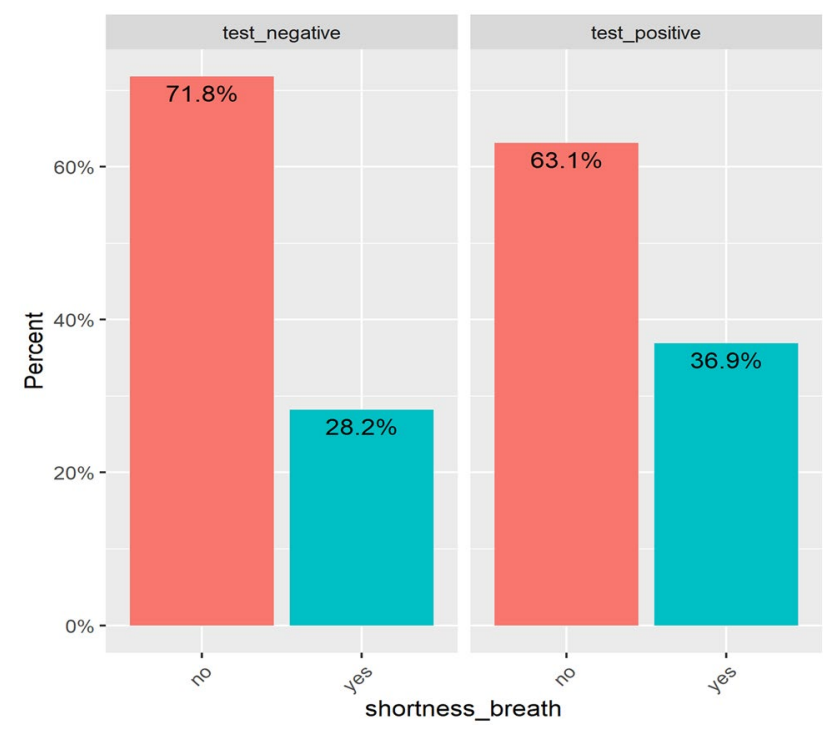

Fig. 3 Self-reported shortness of breath in respondents with a positive and negative COVID-19 test

\section{Fatigue}

Levels of self-reported fatigue were high in both groups with 65/84 (77\%) of COVID-19-positive patients reporting fatigue and 30/39 (77\%) of those who were COVID19-negative reporting fatigue (Fig. 4).

\section{Loss of taste and smell}

New symptoms emerging at the time of data collection included loss of taste and smell. Of our COVID-19-positive

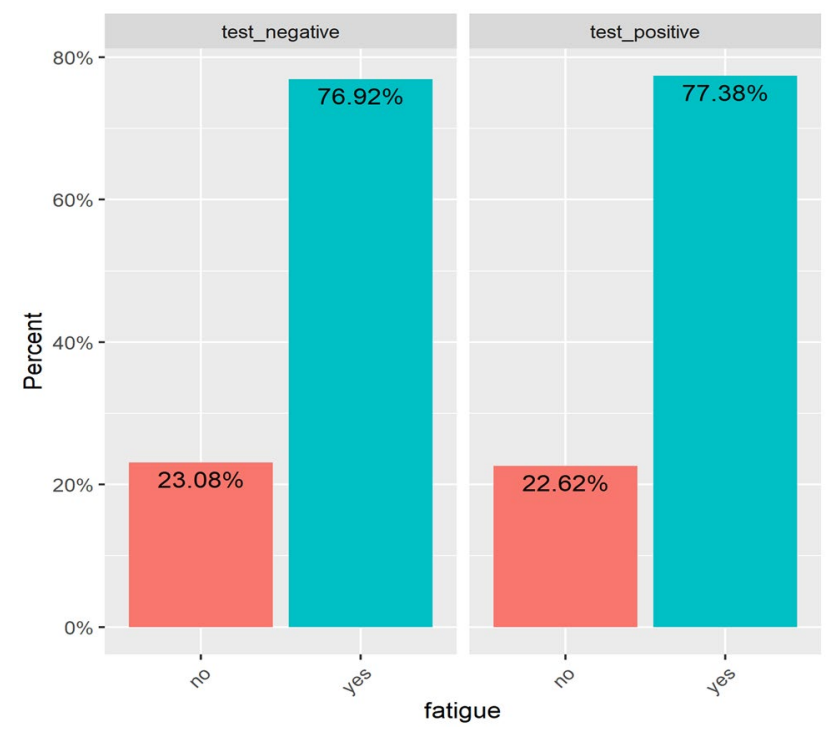

Fig. 4 Self-reported fatigue in respondents with a positive and negative COVID-19 test

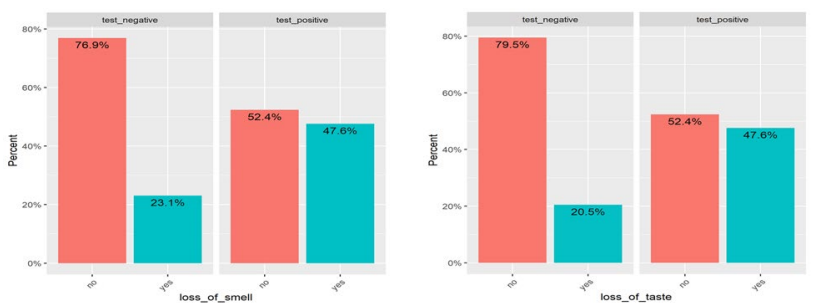

Fig. 5 Self-reported loss of taste and smell in respondents with a positive and negative COVID-19 test

respondents $(n=84), 40(48 \%)$ reported loss of smell and $40(48 \%)$ reported loss of taste. Of those with a negative COVID-19 test, 9 (23\%) reported loss of smell and $8(21 \%)$ reported loss of taste. There was evidence for a higher proportion of loss of smell $(p=0.02)$ and taste $(p=0.01)$ in those reporting a positive result, compared to those reporting a negative result (Fig. 5).

\section{Discussion}

Similar to every country around the world, Ireland has had to adapt to the rapid spread of the COVID-19 pandemic. Previously not detected in humans, SARS-CoV-2 poses unprecedented challenges on a global scale. During the first wave of COVID-19, when the MyCovidSymptoms.ie Web site was established, there remained uncertainty regarding COVID-19's mode of transmission, clinical presentation and pandemic potential [5].

The diagnosis of COVID-19 was complicated by the diversity in symptoms and severity of the disease [6]. Many early case studies were published in an attempt to understand the dynamics of this emerging infection and identify early indicators of disease [7]. However, no such case series described the initial picture of COVID-19 in Ireland.

Table 1 Comparison of symptoms between those reporting a positive and negative test. Statistics presented: median (IQR); $n$ (\%). Statistical tests performed: Wilcoxon rank-sum test for temperature; Fisher's exact test for symptoms

\begin{tabular}{llll}
\hline Symptom & Negative test, $N=39$ & Positive test, $N=84$ & $p$ value \\
\hline $\begin{array}{l}\text { Temperature } \\
\left({ }^{\circ} \mathrm{C}\right)\end{array}$ & $37.75(36.65,38.10)$ & $37.40(36.80,38.20)$ & 0.80 \\
Dry cough & $17(44 \%)$ & $49(58 \%)$ & 0.20 \\
Fatigue & $30(77 \%)$ & $65(77 \%)$ & 0.90 \\
Loss of smell & $9(23 \%)$ & $40(48 \%)$ & 0.02 \\
Loss of taste & $8(21 \%)$ & $40(48 \%)$ & 0.01 \\
$\begin{array}{l}\text { Shortness of } \\
\text { breath }\end{array}$ & $11(28 \%)$ & $31(37 \%)$ & 0.50 \\
\hline
\end{tabular}


Early reports of COVID-19 symptoms identified fever, cough and shortness of breath; these were used for initial case identification and testing prioritisation [8]. While loss of taste and smell are now key features in the recognition of COVID-19, at the time of the Web site launch in April 2020, only anecdotal reports identified these symptoms as possible indicators of disease [9]. During the first wave of the pandemic, those with smell or taste disorders as the initial or main symptom were not suspected to be infected with SARS-CoV-2 and thus were not referred for testing, representing potential routes of transmission of the virus [10].

Our aim was to identify the prevalence of these emerging symptoms, as well as the classical features of COVID-19, in an Irish population. Now, a year into the pandemic, subsequent reports have demonstrated that loss of taste and smell are highly predictive of COVID-19 and are more predictive than the presence of a cough [8].

The COVID-19 pandemic has placed an unprecedented burden on the Irish healthcare system and, in particular, on our capacity for mass testing. Recent studies have shown that the additional criteria of loss of taste and smell, in conjunction with the classical symptoms of COVID-19, can capture up to $95 \%$ of cases of COVID-19 [8]. A more accurate understanding of the clinical picture of COVID-19 allows for more timely testing pathways, which are paramount to rapidly break chains of transmission and prevent generation of secondary and tertiary cases from an Index COVID-19 case [11]. This highlights how applications and Web sites such as MyCovidSymptoms.ie, and other examples of big data and statistical analysis, have emerged globally as a powerful tool in helping with the pandemic.

Similar applications have been used internationally to record self-reported COVID-19 symptoms. An example of this is the COVID Symptom Study app developed by Zoe Global and launched in the UK and the USA in March which offers data on risk factors, predictive symptoms, clinical outcomes and geographical hotspots [4]. These Web-based surveys have been used to harmonise clinical observations to support the development of guidelines and prognostic and diagnostic indicators [12].

In the future, self-reported data from mobile applications may have a role in the detection of rapid case increases and the identification of infection hotspots [13]. In an effort to prevent the need for national restrictions, such data may also be used to identify areas in need of targeted intervention [13]. Data are also used to supplement clinical trials and investigate the long-term effects of COVID-19 [4].

\section{Limitations}

A limitation of this study is that smartphone applications or Web sites do not represent a random sampling of the population and are instead a self-selected group. Another limitation is the self-report nature of the data which could result in false negative and false positive reports being included. Furthermore, there were no data collected on the timing of a test result relative to symptoms.

\section{Conclusion}

These data represents an early picture of the clinical characteristics of COVID-19 in an Irish population. It also highlights the potential use of self-reported data in an attempt to respond to the rapid pace of COVID-19 and aid our effort to mitigate both the current health crisis but also future pandemics.

\section{References}

1. Center CR (2021) COVID-19 Dashboard by the Centre for Systems Science and Engineering: John Hopkins University of Medicine [cited 2021]. Available from: https://coronavirus.jhu.edu/ map.html

2. Centre HPS (2020) Epidemiology of COVID-19 in Ireland. $4 / 12 / 20$

3. Menni C, Valdes AM, Freidin MB et al (2020) Real-time tracking of self-reported symptoms to predict potential COVID-19. Nat Med 26(7):1037-1040

4. Drew DA, Nguyen LH, Steves CJ et al (2020) Rapid implementation of mobile technology for real-time epidemiology of COVID19. Science 368(6497):1362-1367

5. Hu Y, Sun J, Dai Z et al (2020) Prevalence and severity of corona virus disease 2019 (COVID-19): a systematic review and metaanalysis. J Clin Virol 127:104371

6. Guan WJ, Ni ZY, Hu Y et al (2020) Clinical Characteristics of Coronavirus Disease 2019 in China. N Engl J Med. 382(18):1708-20

7. Abed Alah M, Abdeen S, Kehyayan V (2020) The first few cases and fatalities of Corona Virus Disease 2019 (COVID-19) in the Eastern Mediterranean Region of the World Health Organization: a rapid review. J Infect Public Health 13(10):1367-1372

8. Dawson P, Rabold EM, Laws RL et al (2020) Loss of taste and smell as distinguishing symptoms of coronavirus disease 2019 . Clin Infect Dis 72(4):682-685

9. Spinato G, Fabbris C, Polesel J et al (2020) Alterations in smell or taste in mildly symptomatic outpatients with SARS-CoV-2 infection. JAMA 323(20):2089-2090

10. Barillari MR, Bastiani L, Lechien JR et al (2021) A structural equation model to examine the clinical features of mild-to-moderate COVID-19: a multicenter Italian study. J Med Virol 93(2):983-994

11. Ni Bhuachalla C, Murphy N, O'sullivan M et al (2020) COVID19: The Irish public health experience. Ir Med J. 113(7):1-3

12. Bastiani L, Fortunato L, Pieroni S et al (2021) Rapid COVID-19 Screening based on self-reported symptoms: psychometric assessment and validation of the EPICOVID19 Short Diagnostic Scale. J Med Internet Res 23(1):e23897

13. Varsavsky T, Graham MS, Canas LS et al (2020) Detecting COVID-19 infection hotspots in England using largescale self-reported data from a mobile application. medRxiv. 2020.10.26.20219659 